\title{
Parasitismo intestinal y síndrome anémico en preescolares y escolares, San Vicente Centenario, Santa Bárbara, 2014
}

\author{
Tania Soledad Licona Rivera ${ }^{1}$ \\ Silvia Yolanda Acosta Ramírez² \\ Mirna Lizeth Medina Gámez ${ }^{3}$ \\ Roberto Obdulio Tinoco Franzua ${ }^{4}$
}

\section{RESUMEN}

Las infecciones parasitarias son un problema serio en la salud pública, debido a que suelen causar anemia por deficiencia de hierro, mala absorción de nutrientes y diarrea, entre las principales afecciones. En consecuencia, el objetivo de esta investigación era determinar la relación entre parasitismo intestinal y anemia en la población preescolar y escolar de San Vicente Centenario, entre junio y diciembre de 2014.

El estudio es descriptivo correlacional en niños preescolares y escolares de los centros educativos del municipio, se incluyeron a los preescolares y escolares cuyos padres o tutores aceptaron participar, firmando consentimiento informado. Se aplicó un instrumento para obtener información clínica-epidemiológica.

Se realizó hemograma y examen coproparasitológico en un laboratorio clínico de Santa Bárbara. Los casos positivos fueron tratados y se realizaron exámenes de control un mes después. Se utilizó programa SPSS para calcular medidas de tendencia central y pruebas de hipótesis.

\footnotetext{
${ }^{1}$ Beneficiaria de una beca sustantiva de la DICYP, Escuela Universitaria de Ciencias de la Salud, Facultad de Ciencias Médicas, UNAH-Valle de Sula: tania_licona@yahoo.com.mx

2 Profesora auxiliar, Departamento de Educación de Enfermería, Facultad de Ciencias Médicas, UNAH: silvia.acosta@unah.edu.hn

3 Investigadora independiente, monitora en investigación clínica: mirnamedina08@yahoo.com

${ }^{4}$ Investigador independiente, profesor jubilado, UNAH: obduliotinoco@yahoo.com
} 
Los resultados muestran que 351 niños fueron incluidos, de los cuales según el género de los participantes, 183 eran niños (52\%) y 168 (48 \%) niñas; la edad promedio fue 8 años. El 61 \% (214) estaba parasitado; 62 (29\%) con Giardia lamblia, 46 (21.5\%) y con Ascaris lumbricoides tenían infección leve el $17.7 \%$, moderada 37.7 $\%$ y severa $4.5 \%$. De los 102 (77\%) niños menores de 6 años, 46 (45\%) presentaban anemia y de los 249 (71\%) de siete años o más (23\%), ninguno tenía anemia. El total de niños que presentaba anemia leve fue de 103 (29\%), con valores de hemoglobina mayores de $10 \mathrm{~g} / \mathrm{dl}$. Al calcular el coeficiente de Spearman no se encontró correlación entre parásitos y anemia. Se concluye que no se encontró relación entre parasitismo y anemia, pero la prevalencia de parasitismo intestinal es elevada, afectando la salud de los niños.

Palabras claves: amebiasis, anemia, helmintiasis, infecciones por protozoos, parásitos, parasitología.

\section{ABSTRACT}

Parasitic infections are a serious public health problem, because they often cause iron deficiency anemia, malabsorption of nutrients and diarrhea, among others. The objetive of this research was to determine the relationship between intestinal parasitism and anemia in the preschool and school population of San Vicente Centenario, from June to December 2014. The method used was a descriptive correlational study in preschool and school children in the municipality. Only those children whose parents or guardians signed an informed consent agreement were included in this study. An instrument was applied to obtain clinical and epidemiological information.

$\mathrm{CBC}$ and coproparasitological tests were run in a clinical laboratory located in Santa Barbara. Positive cases were treated and control tests were performed one month later. SPSS software was used. Results: 351 children were included. 183 children (52\%) were male and 168 (48\%) were female, their average age was 8 years old. Of all the evaluated children, $214(61 \%)$ were parasitized and $62(29 \%)$ had Giardia lamblia. 46 children $(21.5 \%)$ had different types of Ascaris lumbricoides, in which $17.7 \%$ was mild, $37.7 \%$ was moderate, and $4.5 \%$ was severe. Of the 102 children under 6 years old $(77 \%), 46$ of them $(45 \%)$ had anemia. Of the 249 children over age $7(71 \%)$, only $23 \%$ were not anemic. The 103 children that had anemia was mild type, with hemoglobin levels above $10 \mathrm{~g} / \mathrm{dl}$. When calculating Spearman, no correlation between parasites and anemia was found. Conclusions: Although no relationship 
between parasitism and anemia was found, the prevalence of intestinal parasites is high, thus affecting the health of children.

Keywords: amebiasis, anemia, parasites, helminthiasis, protozoan infection, parasitology. 


\section{INTRODUCCIÓN}

En pleno siglo XXI, la población hondureña en general y los niños en particular, continúan con parásitos intestinales que compiten con su propia vida, ocasionándoles diferentes problemas de salud: síndrome anémico, desnutrición y problemas de aprendizaje, los cuales han sido descritos por Tranieri (2009) y, en el peor de los casos, pueden ocasionar la muerte (Cueto Montoya, 2009). Los factores que predisponen a este problema de salud son diferentes: falta de higiene, educación y servicios básicos (Barón, 2007), aunque según estudios similares, han sido en lugares y tiempo distinto (Zonta, 2007).

Según Bastidas (2012) se estima que 2,000 millones de personas a nivel mundial están en riesgo de enfermar por parasitismo intestinal, 300 millones tienen morbilidad severa asociada y 155,000 mueren anualmente. Con tales cifras, es necesario investigar el tipo de parásitos que afectan la población general de nuestro país, pues lo ideal es saber que especie afecta a cada individuo para poder dar tratamiento efectivo, porque no todas la infecciones de este tipo se tratan de igual manera, incluso se pueden encontrar infecciones por distintos tipos de parásitos.

Con el presente estudio se beneficiará directamente a la población preescolar y escolar del municipio en estudio y se aportará conocimiento científico importante a la literatura hondureña que podrá utilizarse para realizar protocolos de manejo de esta patología. No obstante, incluir a la mayoría de niños fue difícil, pues muchos se ausentaban durante la toma de muestras, pero con la ayuda del personal docente de los diferentes centros educativos fue posible lograr la participación de un alto porcentaje de estudiantes. Esta investigación se realizó con el propósito de determinar la relación existente entre el parasitismo intestinal y la anemia en la población preescolar y escolar del municipio de San Vicente Centenario, Santa Bárbara, en el periodo de junio a diciembre del año 2014.

\section{DEFINICIONES}

Las parasitosis intestinales son un problema de salud pública mundial, especialmente en áreas tropicales y subtropicales. La infección por helmintos es el resultado de la penetración de un gusano al interior del cuerpo en donde maduran, depositan huevos y obtienen nutrición del huésped. Pueden ser provocadas por nematodos intestinales 
o gusanos redondos o por cestodos o gusanos planos. La amebiasis es una infección causada por las amebas Entamoeba dispar y $E$. histolitica. La guiardiasis es la infección causada por la Giardia lamblia. La anemia se define (Tschudy, 2013) como una reducción del volumen de eritrocitos 0 de la concentración de hemoglobina por debajo de los valores normales mínimos para cada edad. En niños de 6 meses a 6 años hay anemia cuando la hemoglobina es inferior a $10.5 \mathrm{~g} / \mathrm{dl}$ y en niños de 7 años 0 más cuando es inferior a $11 \mathrm{~g} / \mathrm{dl}$.

\section{METODOLOGÍA}

Se realizó un estudio de enfoque cuantitativo, alcance correlacional y de tipo transversal en los preescolares y escolares de los centros educativos del municipio San Vicente Centenario del departamento de Santa Bárbara; en los centro de educación básica: Francisco Morazán (364 estudiantes), Jardín de Niños La Primavera (68), Jardín de Niños Mundo Infantil (30), Escuela PROHECO Dagoberto Licona (10), Jardín PROHECO Mi Mundo Feliz (11). El universo se conformó con 483 escolares y preescolares de todos los centros educativos del municipio y la muestra por 351 niños que cumplieron los criterios de inclusión. Inicialmente se socializó el proyecto de investigación con autoridades edilicias y después con los directores, maestros y padres de familia. Se solicitó consentimiento informado a los padres o tutores y asentimiento informado a los niños que cumplían con los criterios de inclusión:

1.Criterios de inclusión:

a. Estudiante activo de uno de los centros educativos del estudio.

b. Cursar el nivel preescolar o escolar hasta sexto grado.

c. Que su padre, madre o tutor brindaran consentimiento informado.

d. Los escolares que sabían leer y escribir brindaran asentimiento informado.

e. Posibilidad del participante de cumplir con todos los procedimientos del estudio según el protocolo.

2.Criterios de exclusión:

a. Haber recibido tratamiento antiparasitario en el último mes.

b. Padecer síndrome anémico de otra etiología.

c. Padecer dolor abdominal agudo el día de inicio del tratamiento.

d. Condición médica que contraindique el uso de antiparasitarios.

e. Hipersensibilidad conocida a alguno de los medicamentos usados en el estudio. 


\section{Procedimientos del estudio}

1. Enrolamiento de participantes: se convocó a padres de familia, maestros y autoridades edilicias, para brindar charlas educativas relacionadas al parasitismo intestinal y se les invitó a participar en el estudio. Una vez que los padres/tutores de los sujetos firmaron el consentimiento, se revisaron los criterios de inclusión y exclusión. Con la ayuda de los padres/tutores y el niño se completó el formulario que incluyó: características generales de los hogares y de la población en estudio, medidas higiénicas-sanitarias, medidas antropométricas (peso y talla), resultados de exámenes de laboratorio (heces fecales, hemograma y ferritina sérica en casos necesarios). Con ayuda de estudiantes de enfermería de la UNAH se compartieron sesiones educativas con los escolares y preescolares sobre la toma correcta de la muestra de heces. Se programó un día por sección y grado para la toma de muestras de heces y sangre. Al recibir los resultados de laboratorio, estos fueron revisados por los médicos investigadores y se brindó tratamiento específico según protocolo (Behrman y otros, 2004).

Cuadro 1. Tratamiento brindado, según protocolo

Helmintos

\begin{tabular}{|c|c|}
\hline Ascaris lumbricoides & $\begin{array}{l}\text { a. Albendazol } 400 \text { mg, dosis única v.o. para todas las edades. } \\
\text { b. Piperazina } 65 \text { mg/kg v.o. cada } 12 \text { horas por } 7 \text { días. }\end{array}$ \\
\hline Trichuris trichura & a. Mebendazol v.o. cada 12 horas por 3 días. \\
\hline Uncinaria & a. Albendazol 400 mg v.o. dosis única para todas las edades. \\
\hline Enterobiasis & $\begin{array}{l}\text { a. Mebendazol } 100 \mathrm{mg} \text { v.o. para todas las edades, repetir dosis } \\
\text { a las } 2 \text { semanas. } \\
\text { b. Tratamiento a familiares. }\end{array}$ \\
\hline Estrongyloidiasis & 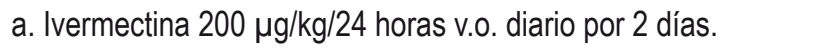 \\
\hline \multicolumn{2}{|l|}{ Protozoos } \\
\hline Amebiasis & a. Metronidazol 30- 50 mg/kg/24 horas dividido v.o. por 10 días. \\
\hline Giardiasis & $\begin{array}{l}\text { a. Metronidazol } 15 \mathrm{mg} / \mathrm{kg} / 24 \text { horas v.0. dividido en tres tomas } \\
\text { por } 5 \text { días, máximo } 750 \mathrm{mg} / 24 \text { horas. }\end{array}$ \\
\hline
\end{tabular}


Para los participantes con parasitosis intestinales, un mes después de tomar el tratamiento antiparasitario, se realizó una nueva toma de muestra de heces para verificar reinfección, en los casos encontrados se dio tratamiento nuevamente.

En los casos que presentaron parásitos y síndrome anémico, se dio el antiparasitario y se instruyó a la madre para que al terminar el antiparasitario continuara con el tratamiento antianémico.

En los niños que presentaron anemia microcítica hipocrómica diagnosticada con volumen corpuscular medio (VCM) y hemoglobina corpuscular media (HCM) por debajo de lo normal, se realizó ferritina sérica para evaluar el grado de deficiencia de hierro y se brindó tratamiento según protocolo: hierro elemental 4-6 mg/kg/día dividido en tres tomas por tres meses.

2. Procedimiento de toma de muestra: se realizaron sesiones educativas sobre cómo tomar la muestra de heces fecales y se les entregó un kit que contenía: instructivo, frasco limpio con tapadera y palillas de madera. La toma de muestra de heces se realizó por defecación espontánea en casa y la muestra de sangre se realizó por punción venosa. Las muestras fueron procesadas en el Laboratorio Paz Sánchez de Santa Bárbara. Se realizaron por día 40 exámenes.

\section{Análisis estadístico}

Los datos fueron tabulados y analizados en el programa de computación SPSS versión 19. Para los datos sociodemográficos de calcularon medidas de tendencia central, para la prueba de hipótesis se calculó el coeficiente de Spearman y el chi-cuadrado de Pearson.

\section{Consideraciones éticas}

Este estudio se realizó de acuerdo a las buenas prácticas clínicas. Se sometió el protocolo al Comité de Ética en Investigación Biomédica de la UNAH para su revisión y aprobación. Se aplicó consentimiento informado a los padres y asentimiento informado a los posibles participantes, en el cual se informó de qué se trataba el estudio, propósito, participación, beneficios y riesgos previos a la participación en el estudio. 


\section{RESULTADOS}

Descripción de la muestra

La muestra utilizada en el estudio fue de 351 niños y niñas de cinco centros educativos de la comunidad de San Vicente, Santa Bárbara, que presenta las siguientes características sociodemográficas:

1. Edad: la edad más frecuentes fue de ocho años (15.7 \%), el promedio de edad de los participantes fue de ocho años, con una desviación estándar de más 0 menos 2.5; el rango de edades fue de catorce, con un máximo de 17 años y un mínimo de 3 años. El 50 \% más joven de los participantes tenía menos de ocho años.

2. Sexo: la distribución por sexo fue 168 (48 \%) niñas y 183 (52\%) niños. Siendo más frecuentes los niños.

3. Centro educativo al que pertenecían y grado que cursaban: CEB Francisco Morazán: 276 niños (primer grado, 43; segundo, 59; tercero, 56; cuarto, 47; quinto, 42 y sexto, 29). Jardín La Primavera: 35 niños (prekínder: 4, kínder: 11 y preparatoria: 20). Jardín Mundo Infantil: 27 niños (prekínder, 2; kínder, 11 y preparatoria, 14). Escuela Dagoberto Licona: 9 niños de primer grado. Jardín Mi Mundo Feliz: 4 niños de kínder.

4. Hábitos y condiciones de vida: se encontró que 346 (98.6\%) de los niños se lavaban las manos antes de comer y después de ir al baño. $229(65.2 \%)$ tomaban agua de tubería, $24(6.8 \%)$ de pozo y $96(27.4 \%)$ de botellón. El tratamiento que le dan al agua antes de consumirla: $84(23.9 \%)$ la hierven, $32(9.1 \%)$ la cloran, $87(24.8 \%)$ la filtran, $141(40.2 \%)$, la ingieren sin tratarla y 7 no contestaron. $42(12 \%)$ de los niños realizan la disposición de excretas al aire libre, los demás lo hacen en servicio lavable o letrina. Se encontró que 125 (35.6\%) acostumbran a andar descalzos, 60 (17.1\%) suelen jugar tierra y $64(18.2 \%)$ se comen las uñas. $212(60.4 \%)$ de los niños viven en hacinamiento (3 o más personas por habitación).

5. Parásitos: del total de niños estudiados, 137 (39 \%) no tenían parásitos y 214 (61 \%) estaban parasitados. De los 214 niños parasitados, 198 tenían parásitos patógenos y el resto poseían parásitos comensales o no patógenos únicamente. 
Los parásitos patógenos más frecuentemente encontrados fueron las amebas; 62 niños (17.7 \%) con Guiardia lamblia, seguido de los helmintos; 46 (13.1\%) con Áscaris lumbricoides, 37 (10.5\%) con Trichuris trichura y 27 (7.7\%) resultaron multiparasitados; siendo la Guiardia lamblia el parasito más encontrado (ver gráfico 1).

Gráfico 1. Tipo de parásitos presentes en los escolares

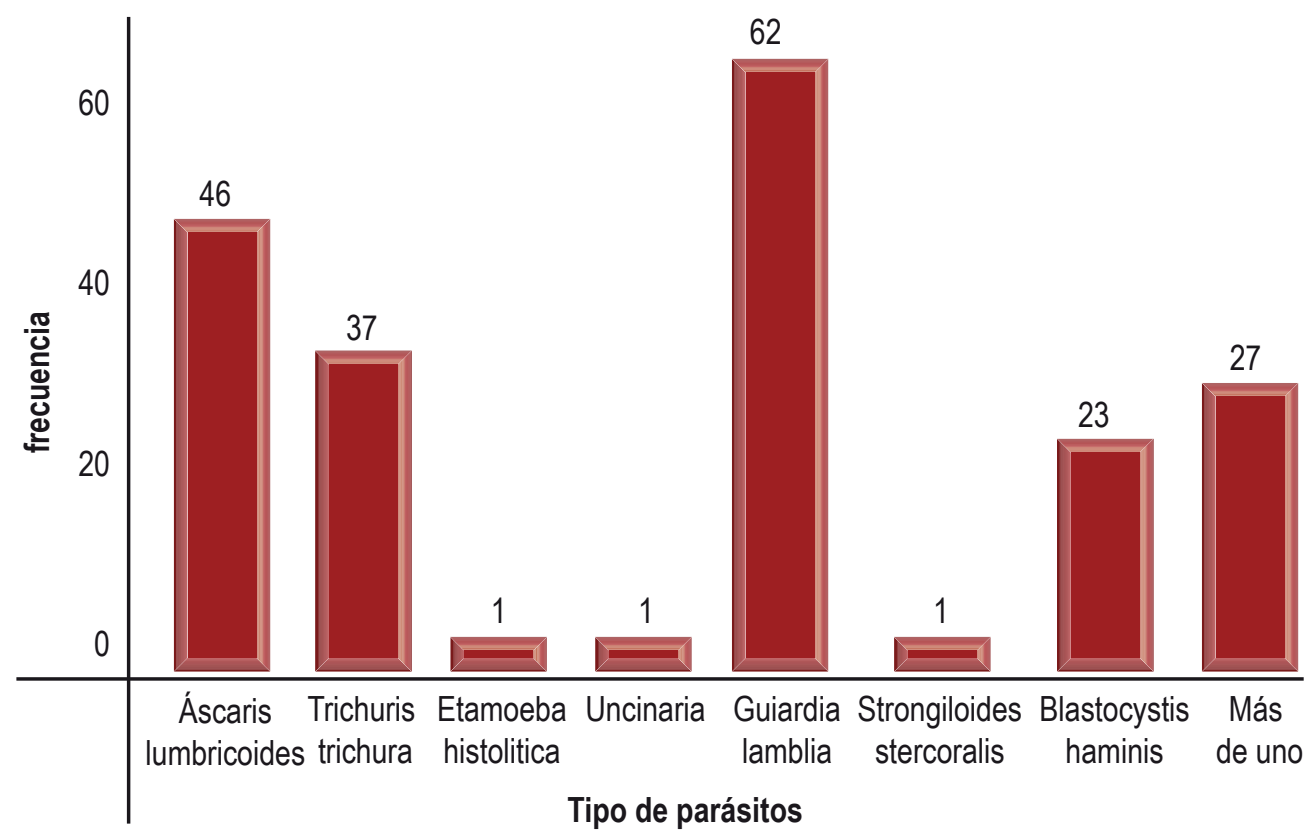

Debido a que el examen general de heces (EGH) en algunas ocasiones reporta que no hay parásitos aun con la presencia de estos, a todos los niños se les realizó, además de este, un examen con una técnica especial llamado concentrado de heces, resultando positivo en 87 niños (detectando Áscaris lumbricoides y Trichuris trichura), a pesar de que en el EGH no se habían detectado.

De los 46 pacientes que presentaban Áscaris lumbricoides, 18 fueron diagnosticados por el examen concentrado de heces (no brinda cantidad de huevos) y el grado de infección fue moderado en la mayoría de los casos (37.7 \%) y severo en dos casos (4.4\%). Ver gráfico 2. 
Gráfico 2. Grado de infección en pacientes con Áscaris lumbricoides

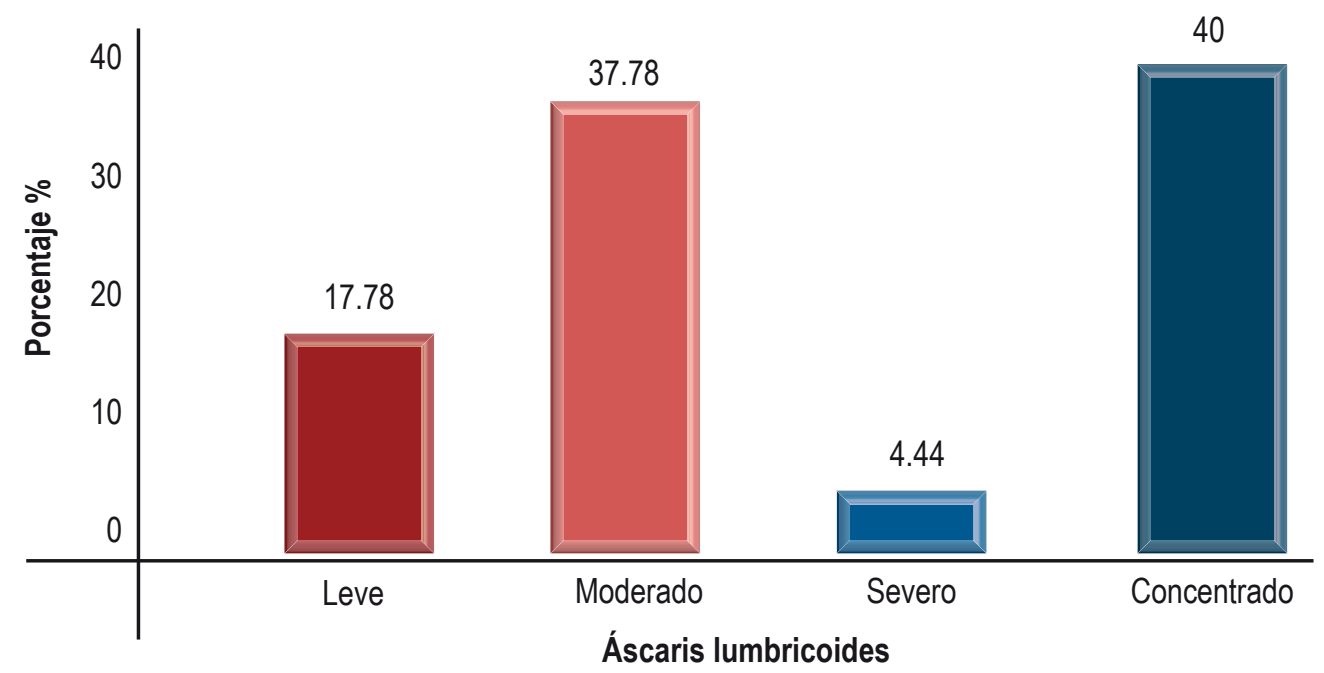

De los 62 pacientes que tenían Trichuris trichura, 37 tenían Trichuris trichura únicamente y 27 tenían Trichuris trichura combinados con otros parásitos; en 50 (80.6\%) el diagnóstico se realizó mediante el examen concentrado de heces (no brinda el número de huevos encontrados) y en ningún caso hubo infección severa (ver gráfico 3).

Gráfico 3. Grado de infección por Trichuris trichura

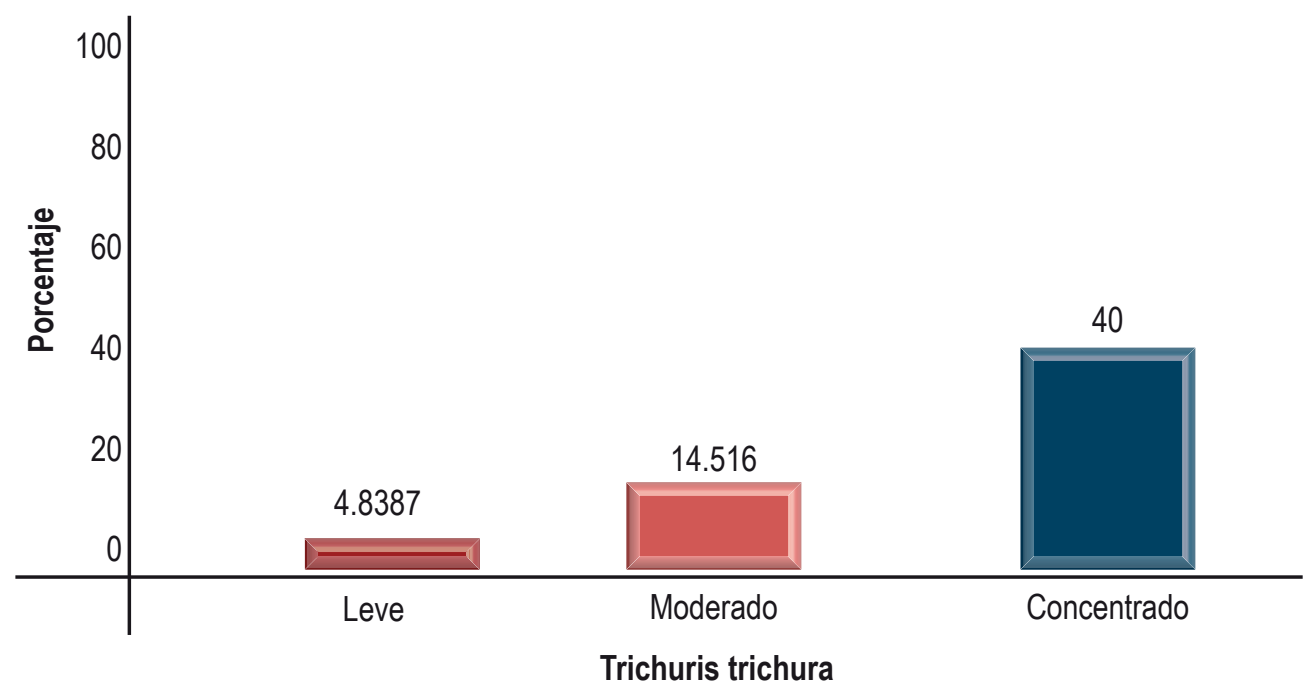


6. Anemia: se encontró que $45.1 \%$ de los niños de seis años o menos presentaban anemia, comparado con el $22.9 \%$ en los de siete años o más (ver tabla 1). 103 (29.3\%) de los niños tenían anemia, siendo de tipo microcítica hipocrómica en 95 de los casos. Se realizó ferritina sérica en cuatro de los casos, las cuales resultaron normales.

7. Anemia según grupos de edad: de los 103 niños con anemia, 46 (44.7 \%) tenían 6 años o menos y 57 (55.3 \%) tenían 7 años o más. Con relación al número de niños en cada grupo, de los 102 niños de 6 años o menos, 46 (45.1\%) tenían anemia y de los 249 de 7 años o más, 57 (22.9\%) presentaban anemia.

8. Anemia y presencia de parásitos en niños preescolares y escolares: se encontró que 35 de los 66 preescolares (53\%) tenían anemia y 46 (70\%) presentaban parásitos (ver tabla 1). Se observa que los niños preescolares presentan más anemia y parásitos.

Tabla 1. Anemia y presencia de parásitos en los niños preescolares y escolares

\begin{tabular}{|l|c|c|c|}
\hline \multicolumn{1}{|c|}{ Patología } & & & \\
\hline Nivel educativo & Total de niños & Anemia & Parasitismo \\
\hline Preescolares & 66 & $35(53 \%)$ & $46(70 \%)$ \\
Escolares & 285 & $68(24 \%)$ & $168(59 \%)$ \\
Total & 351 & 103 & 214 \\
\hline
\end{tabular}

9. Exámenes control: a los 214 niños que se encontraron con parásitos, se les brindó tratamiento médico según el tipo de parásitos encontrados. Un mes después fueron citados nuevamente para realizarles exámenes de control, presentándose 155 de ellos, encontrando que 88 (57.32\%) estaban sin parásitos y $67(42.68 \%$ ) todavía los tenían (ver gráfico 4). De los 103 niños con anemia, $40(39 \%)$ se presentaron a la realización del hemograma control, encontrándose que 31 (77.5 \%) de ellos ya no tenían anemia, ya que había aumentado considerablemente su hemoglobina. 
Gráfico 4. Presencia de parásitos en el examen de heces control

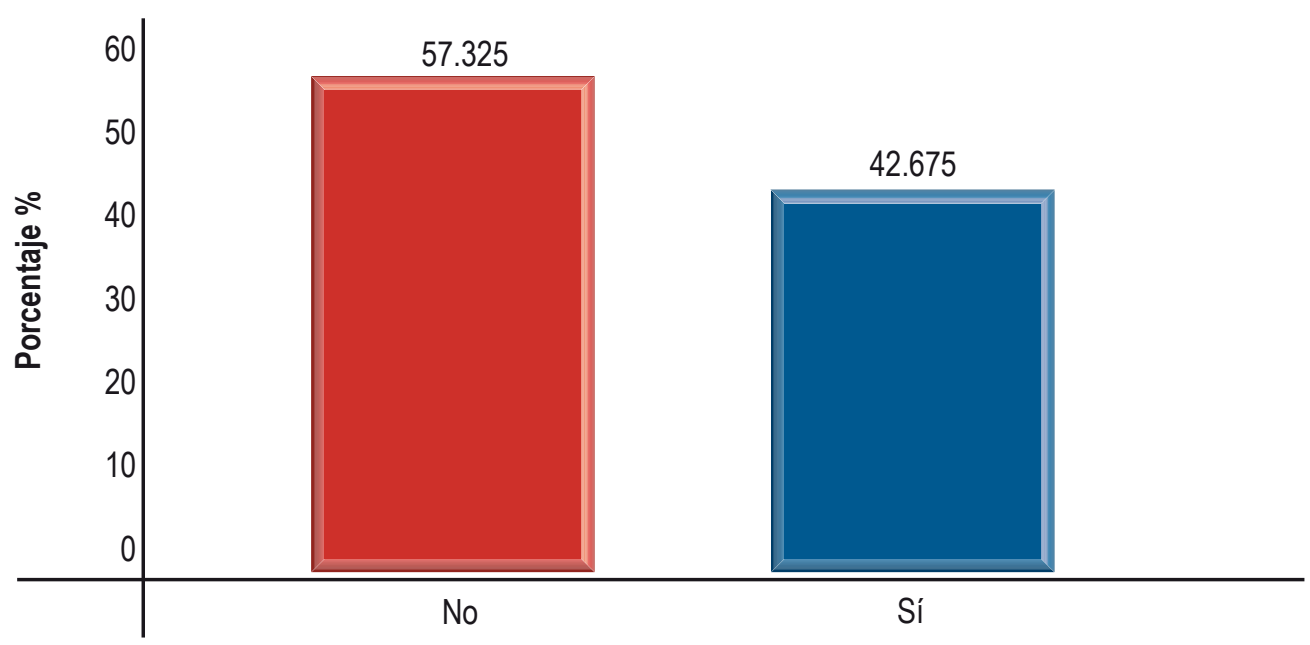

Presencia de parásitos $C$

\section{Prueba de hipótesis}

1. H1. A menor edad, mayor grado de infección parasitaria intestinal: se encontró que no existe correlación entre la edad y el grado de infección parasitaria, el coeficiente de Spearman $=-0.046$, el valor de $P=0.505$ ( $P$ mayor que 0.05). Lo cual indica que la correlación no es estadísticamente significativa.

2. H2. A mayor grado de infección parasitaria, menor concentración de hemoglobina: no se encontró correlación entre el grado de infección parasitaria y la concentración de hemoglobina. El coeficiente de Spearman, Rho= -0.080, $P=0.244(P$ mayor que 0.05), lo que significa que no es estadísticamente significativo.

3. H3. A mayor grado de infección parasitaria intestinal, mayor presencia de anemia: según la evidencia mostrada en la tabla 1 , se observa que el coeficiente de Spearman Rho=0.112 y $P=0.102$ (P mayor que 0.05), lo cual significa que la correlación es estadísticamente no significativa.

4. H4. Existe relación positiva entre parasitismo intestinal y anemia: no se encontró relación entre parasitismo intestinal y anemia. En el chi cuadrado de Pearson $=0.592$, el valor de $P=0.442$ ( $P$ mayor que 0.05 ), lo cual significa que no existe relación. 


\section{DISCUSIÓN}

Frecuentemente, la elevada prevalencia de parasitosis está relacionada con la contaminación fecal del suelo y agua de consumo o de los alimentos, unida a deficientes condiciones sanitarias y socioculturales. Específicamente, la Trichuris trichiura es mucho más frecuente en las comunidades rurales en condiciones sanitarias inapropiadas y suelos contaminados por heces animales o humanas y la $\mathrm{E}$. histolytica se transmite por alimentos o agua contaminada con heces fecales.

Por tales motivos, es de suma importancia indagar sobre los hábitos higiénicossanitarios y condición socioeconómica de la población en estudio, en este caso se encontró que la fuente de obtención del agua para consumo y el tratamiento que le dan a esta, se relaciona con la presencia de parasitismo intestinal en los participantes, pues solamente el $59 \%$ de la población trata el agua que ingiere y la principal fuente de abastecimiento es la tubería; una minoría (27.4\%) consume agua embotellada.

Hay un porcentaje considerable (12\%) de familias que hacen sus necesidades fisiológicas al aire libre, lo que puede contaminar las fuentes de agua, esta necesidad insatisfecha sumada a otras condiciones socioeconómicas desfavorables, está contribuyendo a las parasitosis intestinales. Otro factor es el hacinamiento entre los participantes, que es alto, ya que el $60 \%$ de las familias viven en esta situación y el $8 \%$ de la población en estudio tienen piso de tierra.

Una de las actividades realizadas como parte del estudio, fue el proyecto que se denominó "Si a los parásitos quieres matar, las manos te debes lavar", este fue desarrollado por estudiantes de la Carrera de Enfermería de la UNAH y abarcó a los 5 centros educativos participantes. Debido a este proyecto, el $90 \%$ manifiesta lavar sus manos en diferentes momentos: antes de ingerir los alimentos, después de ir al baño y después del contacto con animales, práctica que se espera mantener para que contribuya a disminuir el parasitismo intestinal.

Jugar con tierra o comerse las uñas no son prácticas comunes entre los participantes del estudio, menos del $20 \%$ lo hacen. Andar descalzo es la práctica más frecuente, con un $36 \%$.

En Caldas, Colombia, en una investigación realizada por Cardona Arias y otros (2014), se encontró que el hacinamiento domiciliario afecta al $35 \%$ de las familias, sin 
embargo, en esta investigación se encontró que más de la mitad de los niños (60.4 \%) viven en hacinamiento.

En un estudio realizado en Costa Rica por Abrahams Sandí y otros (2005), se observó positividad por organismos parásitos y comensales del 47,6\%; en contraste con esta investigación en donde se encontró que el $61 \%$ de los niños estaban parasitados.

En Honduras, Kaminsky y otros (1998) realizaron una investigación en diferentes poblaciones, encontrando que en niños de hogar temporal, la prevalencia de parásitos intestinales de 106 niños se diagnosticaron 33 (31\%) infecciones por Ascaris lumbricoides, de las cuales 5 tenían cuenta de huevos mayor de 100/2 mg 25 (23.5 \%) por Trichuris trichiura, todas leves; 14 (13.2 \%) por Strongiloides stercoralis; 3 (2.8\%) por Himenolepis nana; 28 (26.4\%) por Guiardia lamblia y 6 (6 \%) por E. histoly tica/E. dispar.

G. lamblia estuvo presente en niños 0-11 meses (2 casos, $8 \%$ ), difiriendo de los resultados de este estudio, en el cual se encontró que los parásitos más frecuentemente encontrados son Guiardia lamblia en 62 niños (17.7 \%), seguido de Áscaris lumbricoides 46 (13.1\%) y Trichuris trichura en 37 (10.5\%) niños. Además, se encontró que la intensidad de la infección por Áscaris lumbricoides fue severa en un $5 \%$ de los casos y para Trichuris trichura no se encontró ningún caso severo.

En otra investigación realizada por Kaminsky (2012) en el Hospital de Tela, se encontró que las infecciones por $A$. lumbricoides fueron del $14.5 \%$, entre las cuales un paciente presentaba ascaridiasis severa; también se encontraron 64 infecciones (8.8\%) por T. trichiura, de las cuales había una tricuriasis severa $(72 \mathrm{~h} / 2 \mathrm{mg})$ y se informó también una uncinariasis $(0.7 \%)$, con menos frecuencia; $5.4 \%$ de las muestras resultó positivo por quistes de $\mathrm{G}$. lamblia. En contraste con los resultados de esta investigación, aquí predominaron los protozoos, específicamente Guiardia lamblia. Además, se encontró un niño parasitado por uncinarias. 


\section{CONCLUSIONES}

1. La prevalencia de parasitismo intestinal en la población preescolar y escolar de los centros educativos de San Vicente Centenario, Santa Bárbara, en el periodo de junio a diciembre de 2014 fue del $61 \%$.

2. Las especies parasitarias que afectan a la población preescolar y escolar de los centros educativos de San Vicente Centenario son, en orden de importancia: Giardia lamblia, Áscaris lumbricoides, Trichuris tichiura, Blastocystis hominis, Uncinaria, Taenia solium y Entamoeba histolytica.

3. El grado de infestación por Áscaris lumbricodes y Trichuris trichura en la población preescolar y escolar de los centros educativos de San Vicente Centenario en la mayoría de los casos es moderado.

4. El grupo etario de 6 y menos años es el más afectado por las parasitosis intestinales en los centros educativos de San Vicente Centenario.

5. Los factores relacionados o predisponentes a las parasitosis intestinales que tienen los preescolares y escolares de San Vicente Centenarios son: falta de tratamiento o calidad del agua para consumo, seguridad de la fuente de abastecimiento del agua, eliminación inadecuada de excretas, hacinamiento y piso de tierra.

6. Los hábitos relacionados con las parasitosis intestinales que practican los preescolares y escolares de San Vicente Centenarios son: jugar con tierra, comerse las uñas y andar descalzos. La prevalencia de anemia en la población preescolar y escolar de los centros educativos de San Vicente Centenario, Santa Bárbara, en el periodo de junio a diciembre de 2014 fue del $29.3 \%$.

7. La anemia microcitica hipocrómica es la más frecuente entre los preescolares y escolares con anemia de los centros educativos de San Vicente Centenario. No se encontró relación entre parasitismo intestinal y anemia. 


\section{RECOMENDACIONES}

A la comunidad científica nacional

1. Que se utilicen estos resultados como línea de base para realizar estudios relacionados al tema de parasitismo/anemia y sus consecuencias en preescolares y escolares.

2. Los resultados obtenidos pueden ser utilizados para análisis más profundos por parte de expertos en varias ramas de la medicina.

A las autoridades edilicias municipales

3. Que los resultados obtenidos en el estudio sirvan de insumo para gestionar proyectos de mejora en los centros educativos participantes.

4. Que las autoridades locales gestionen proyectos de mejora de las viviendas encontradas en situación desfavorable.

A los maestros de los centros educativos

5. Que los maestros continúen cultivando las buenas prácticas higiénicas-sanitarias entre los preescolares y escolares para que mantengan y mejoren su salud.

6. Dar seguimiento, conjuntamente con las autoridades locales, a todos los preescolares y escolares que se encontraron parasitados o con anemia.

A la Secretaría de Salud

7. Considerar el uso de antiparasitarios de amplio espectro cuando se realicen campañas masivas de desparasitación, pues según los resultados obtenidos, los niños además de ser afectados por helmintos también presentan protozoos. 


\section{AGRADECIMIENTO}

A la DICYP de la UNAH, por brindarnos una beca sustantiva de investigación para realizar este proyecto.

\section{BIBLIOGRAFÍA}

Abrahams Sandí, Elizabeth; Solano, Mayra y Rodríguez, Beatriz. (2005). Prevalencia de parásitos intestinales en escolares de Limón Centro, Costa Rica. Revista Costarricense de Ciencias Médicas, 26(1-2). Recuperado de: http://www.scielo.sa.cr/scielo.php?script=sci_arttext\&pid=S0253-29482005000100004\&Ing=es

Baron, M. A.; Solano, R. L.; Páez, M.C. y Pabón, M. (2007). Estado nutricional de hierro y parasitosis intestinal en niños de Valencia, Estado Carabobo, Venezuela. Anales Venezolanos de Nutrición, 20(1), 5-11. Recuperado de: http://www.scielo.org.ve/scielo.php?pid=S0798-07522007000100002\&script=sci_arttext

Bastidas, Gilberto; Rojas, Carolina; Martínez Silva, Elisa; Loaiza, Lisbeth; Guzmán, María y otros. (2012). Prevalencia de parásitos intestinales en manipuladores de alimentos en una comunidad rural de Cojedes, Venezuela. Acta Médica Costarricense. Recuperado de: http://www.scielo.sa.cr/scielo.php?script=sci_arttext\&pi$d=S 0001-60022012000400007 \&$ Ing $=e s$

Behrman, Richard E.; Kliegman, Robert M. y Jenson Hal, B. (2004). Tratado de pediatría. España: Elsevier.

Cardona Arias, Jaibert Antonio; Rivera Palomino, Yennifer; Carmona Fonseca, Jaime. (2014). Salud indígena en el siglo XXI: parásitos intestinales, desnutrición, anemia y condiciones de vida en niños del resguardo indígena Cañamomo-Lomaprieta, Caldas, Colombia. Médicas UIS, 27(2), 29-39. Recuperado de: http://www.scielo.org.co/scielo.php?script=sci_arttext\&pi$d=S 0121-03192014000200004 \&$ Ing=en\&tlng=es

Cueto Montoya, Gladys Antonia; Pérez Cueto, María del Carmen; Mildestein Verdés, Silvia; Núñez Linares, María Elena; Alegret Rodríguez, Milagros; Martínez Flores, Nilda. (2009). Características del parasitismo intestinal en niños de dos comunidades del policlínico "XX Aniversario". Revista Cubana Medicina General Integral, 25(1). Recuperado de: http://scielo.sld.cu/scielo.php?script=sci_arttext\&pid=S0864-21252009000100008\&lng=es 
Kaminsky, Rina y otros. (1998). Prevalecía de parasitismo intestinal en diferentes poblaciones de Honduras. Niños y adultos institucionalizados. Revista Médica Hondureña, 66(2), 62-70.

Kaminsky, Rina. (2012). Aspectos epidemiológicos y conceptuales de parasitosis intestinales en el Hospital Regional de Tela, Honduras. Revista Médica. Hondureña 80, (3), 90-95.

Tschudy Megan, M.; Arcada Kristin, M. (2013). Manual Harriet lane de Pediatría. Barcelona: Elsevier.

Tranieri, Mirna; Silva, Ivana; Molina, Yanira; Monges, Darai; Montenegro, Leiby; Morales, Miguel y otros. (2009). Parasitosis intestinales en alumnos de la Unidad Educativa Carabobo: Belén, Municipio Carlos Arvelo, Estado Carabobo, Venezuela. Comunidad y Salud, 7(1), 23-28. Recuperado de: http://www2.scielo.org.ve/scielo.php?script=sci_arttext\&pid=S1690-32932009000100005\&Ing=es

Zonta, María Lorena; Navone, Graciela Teresa; Oyhenart, Evelia Edith. (2007). Parasitosis intestinales en niños de edad preescolar y escolar: situación actual en poblaciones urbanas, periurbanas y rurales en Brandsen, Buenos Aires, Argentina. Parasitología Latinoamericana, 62(1-2), 54-60. Recuperado de: http://dx.doi.org/10.4067/S0717-77122007000100009 